\title{
Soft QCD measurements in the forward acceptance at the $\mathrm{LHC}$
}

Michael Schmelling - MPI for Nuclear Physics

- on behalf of the LHCb collaboration -

\section{Outline}

$\square$ The LHCb experiment

$\square$ Energy flow and multiplicities

- Identified particles

- Drell-Yan production

a Summary and outlook

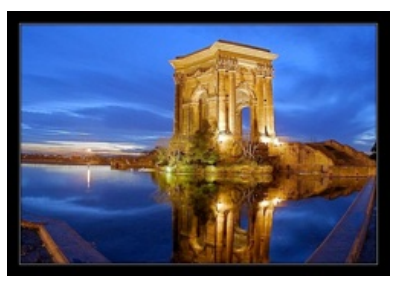

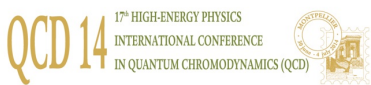




\section{LHC.p 1. THE LHCb EXPERIMENT}

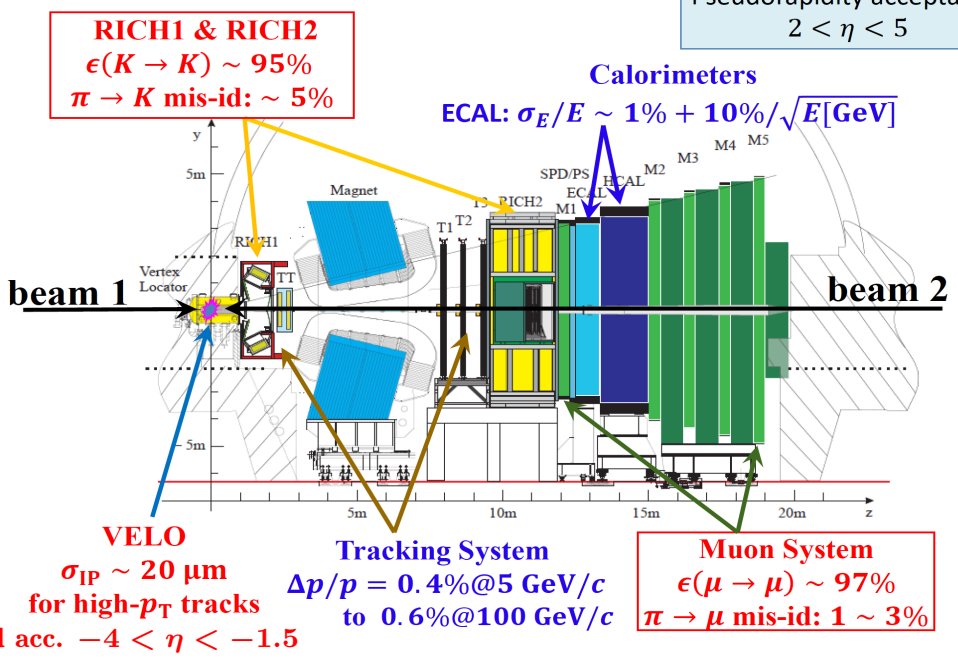




\section{wнсы Data taking history}

LHCb Integrated Luminosity

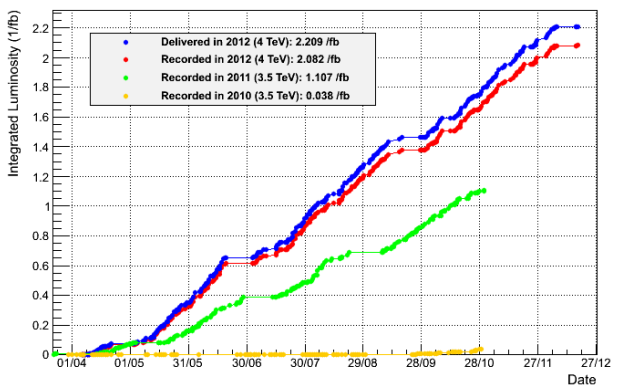

$\begin{array}{ccl}\text { year } & \text { int.luminosity } & E[T e V] \\ 2009 & 6.8 \mu \mathrm{b}^{-1} & 0.9 \\ 2010 & 0.3 \mathrm{nb}^{-1} & 0.9 \\ 2010 & 37 \mathrm{pb}^{-1} & 7 \\ 2011 & 0.1 \mathrm{pb}^{-1} & 2.76 \\ 2011 & 1 \mathrm{fb}^{-1} & 7 \\ 2012 & 2 \mathrm{fb}^{-1} & 8 \\ 2013 & 1.3 \mathrm{nb}^{-1} & 5(\mathrm{pA}) \\ 2013 & 0.6 \mathrm{nb}^{-1} & 5(\mathrm{Ap}) \\ 2013 & 3.3 \mathrm{pb}^{-1} & 2.76\end{array}$

data taking efficiency $93 \%$

$\square$ instantaneous luminosity up to $L=4 \times 10^{32} \mathrm{~cm}^{-2} \mathrm{~s}^{-1}$

$\rightarrow$ twice design value at double the nominal bunch spacing

$\rightarrow$ luminosity leveling for $\mathrm{LHCb}$ by beam steering

$\square$ a total of about $2 \times 10^{14} \mathrm{pp}$-collisions scrutinized 


\section{LAGed 2. ENERGY FLOW AND MULTIPLICITIES}

$\rightarrow$ Energy Flow (EF): average energy per event in a given $\eta$-interval

$$
E F: \quad \frac{1}{N_{\text {int }}} \frac{d E}{d \eta}=\frac{1}{\Delta \eta}\left(\frac{1}{N_{\text {int }}} \sum_{i=1}^{N_{\text {part }, \eta}} E_{i, \eta}\right)
$$

$\rightarrow$ part of underlying event

$\rightarrow$ sensitive to multi-parton interactions \& parton radiation

$\rightarrow$ comparison to PYTHIA 6, PYTHIA 8 and cosmic ray models (generators used to model cosmic ray induced air showers)

analysis for different event classes:

$\square$ inclusive minimum bias:

$\square$ hard scattering: inclusive $\& \& \geq 1$ tracks with $p_{T}>3 \mathrm{GeV} / \mathrm{c}$

$\square$ diffractive enriched: inclusive \&\& 0 tracks with $\eta \in[-3.5,-1.5]$

$\square$ non-diffractive enriched: inclusive \&\& $\geq 1$ tracks with $\eta \in[-3.5,-1.5]$ 


\section{whch Energy Flow: data vs PYTHIA}

$\rightarrow$ total $E F=($ charged + neutral $) E F$

$\square$ Energy Flow increases with momentum transfer $E F_{\text {diff }}<E F_{\text {incl }}<E F_{\text {ndiff }}<E F_{\text {hard }}$

$\square$ highest sensitivity at large $\eta$

$\square$ uncertainties:

$\rightarrow$ dominated by systematics

$\rightarrow$ smallest at large $\eta$

PYTHIA 6: Energy Flow is

$>$ overestimated at small $\eta$

$>$ underestimated at large $\eta$

PYTHIA 8.135 default:

$>$ except for hard scattering the Energy Flow is well described for all samples
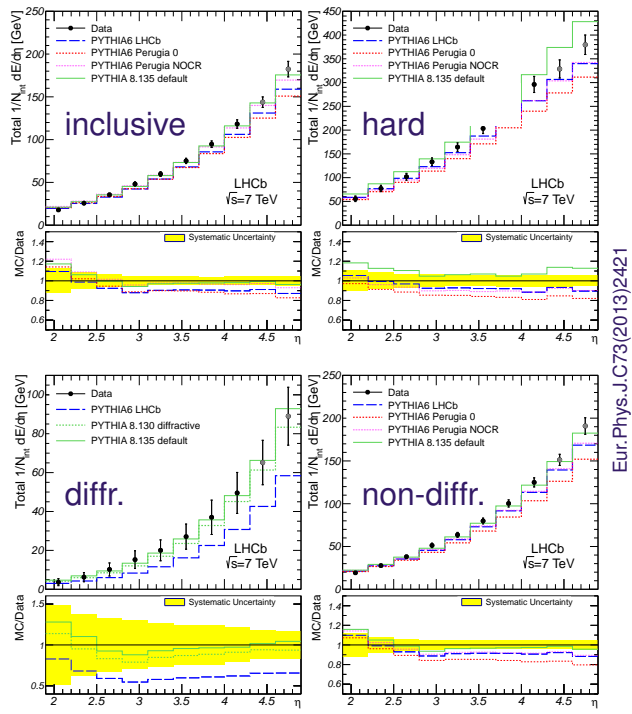


\section{whch Energy Flow: data vs cosmic ray models}

$\rightarrow$ models not tuned to $\mathrm{LHC}(b)$

$>$ EPOS \& SIBYLL: good description of EF for inclusive and non-diffractive events

$>$ QGSJET models: overestimated EF for inclusive and non-diffractive events; good description of hard scattering

$>$ best description by SIBYLL

$>$ all models underestimate the EF of diffractive events
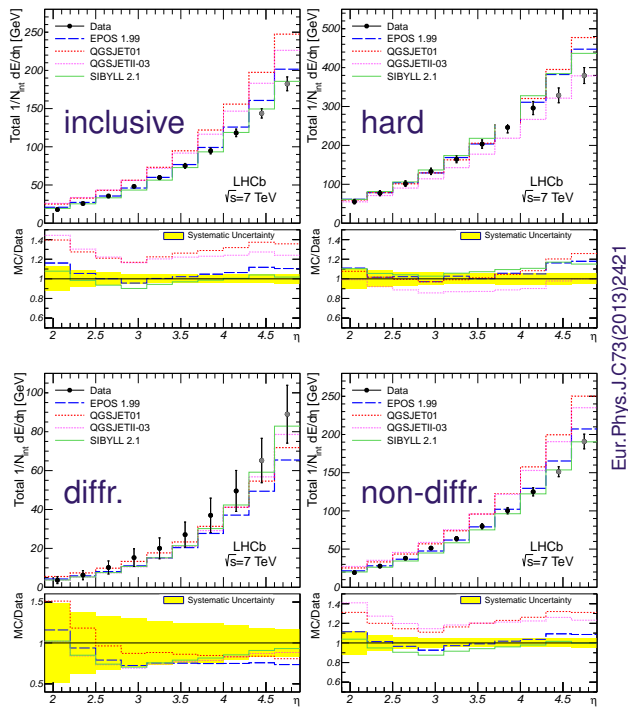


\section{LHCh Charged particle multiplicities}

$\rightarrow$ multiplicity measurement with momentum information

$\square$ kinematics: $p_{T}>0.2 \mathrm{GeV} / c, p>2 \mathrm{GeV} / c, 2.0<\eta<4.8$

low-pileup $(<4 \%)$ minimum-bias pp sample at $\sqrt{s}=7 \mathrm{TeV}$

$\square$ systematic uncertainties $1-10 \%$ - dominant contribution from dead material

charged particle density per event vs $\eta$
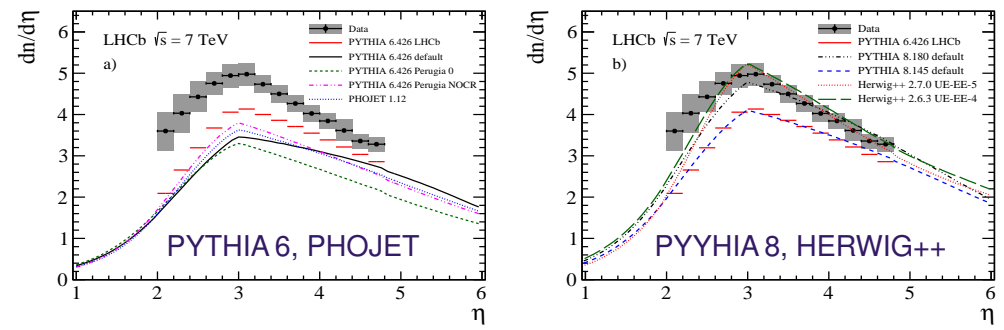

$\rightarrow$ PYTHIA 6 and PHOJET (not tuned to LHC) underestimate densities

$\rightarrow$ satisfactory description by PYTHIA 8.180 (LHC tune) and HERWIG++

$\rightarrow$ best models underestimate density at low $\eta$ and overestimate at large $\eta$ 


\section{whch Particle densities (cont'd)}

charged particle density per event vs $p_{T}$

$\square$ kinematics: $p>2 \mathrm{GeV} / c, 2.0<\eta<4.8$
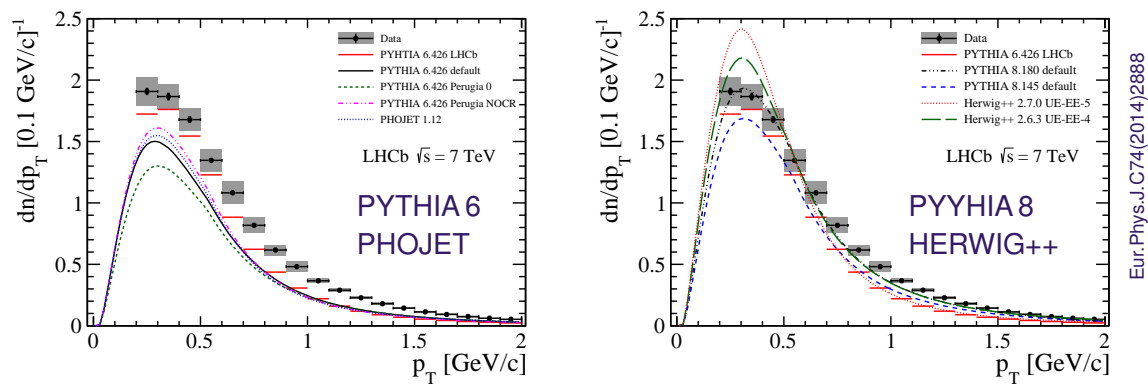

$\rightarrow$ most PYTHIA 6 models and PHOJET too low, but shape OK

$\rightarrow$ too soft distribution for PYTHIA 6 LHCb-tune

$\rightarrow$ reasonable description by PYTHIA 8.180

$\rightarrow$ normalization OK but too soft spectrum for HERWIG++ 


\section{Lucb Multiplicity distributions}

$\square$ kinematics: $p>2 \mathrm{GeV} / c, p_{T}>0.2 \mathrm{GeV} / c, 2.0<\eta<4.8$
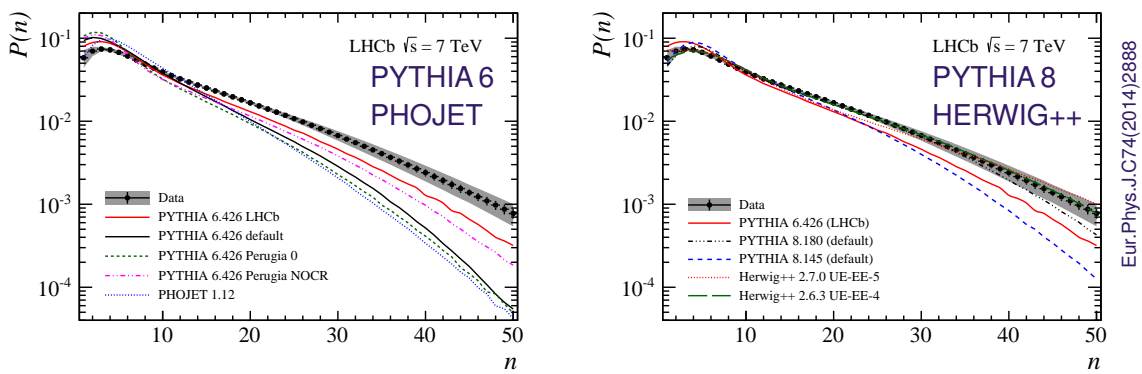

$\rightarrow$ too few charged particles in PHOJET and PYTHIA 6 tunes

$\rightarrow$ PYTHIA 8.180 and HERWIG++ (tuned to central LHC data) do best

$\rightarrow$ none of the models studied is fully able to describe all distributions 


\section{Inch 3. IDENTIFIED PARTICLES}

$\rightarrow$ particle production ratios as a function of $y$ and $p_{T}$

$\square$ antiparticle/particle ratios and ratios of different particle species

$$
\frac{\pi^{-}}{\pi^{+}}, \frac{K^{-}}{K^{+}}, \frac{\bar{p}}{p}, \frac{\bar{\Lambda}}{\Lambda} \text { and } \frac{K^{+}+K^{-}}{\pi^{+}+\pi^{-}}, \frac{p+\bar{p}}{\pi^{+}+\pi^{-}}, \frac{p+\bar{p}}{K^{+}+K^{-}}, \frac{\bar{\Lambda}}{K_{S}^{0}}
$$

$\square$ many systematic uncertainties cancel

$\square$ mainly information about the hadronization process:

$\rightarrow$ baryon number transport from $\bar{p} / p$ and $\bar{\Lambda} / \Lambda$

$\rightarrow$ baryon suppression from baryon/meson ratios

$\rightarrow$ strangeness suppression from kaon/pion ratios

* experimental aspects:

$>$ results based on $0.3 \mathrm{nb}^{-1}$ at $\sqrt{s}=0.9 \mathrm{TeV}$ and $1.8 \mathrm{nb}^{-1}$ at $\sqrt{s}=7 \mathrm{TeV}$

$>$ PID efficiencies from $K_{S}^{0} \rightarrow \pi^{+} \pi^{-}, \Lambda \rightarrow p \pi^{-}, \bar{\Lambda} \rightarrow \bar{p} \pi^{+}$and $\phi \rightarrow K^{+} K^{-}$

$>$ dominant uncertainties from PID due to limited size of calibration sample 


\section{LHCb Antiparticle/particle ratios}

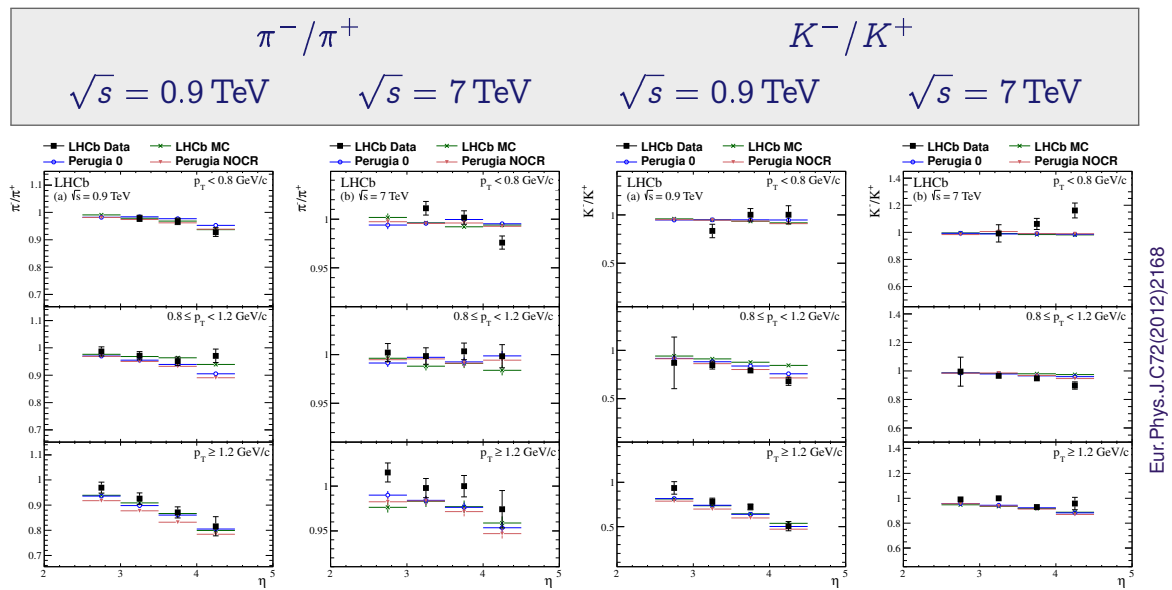

$\rightarrow$ charge ratio drops towards larger rapidities (proton beam)

$\rightarrow$ effect more pronounced at higher $p_{T}$

$\rightarrow$ general behavior reproduced by all PYTHIA 6 tunes 


\section{LHch Strangeness and baryon suppression}

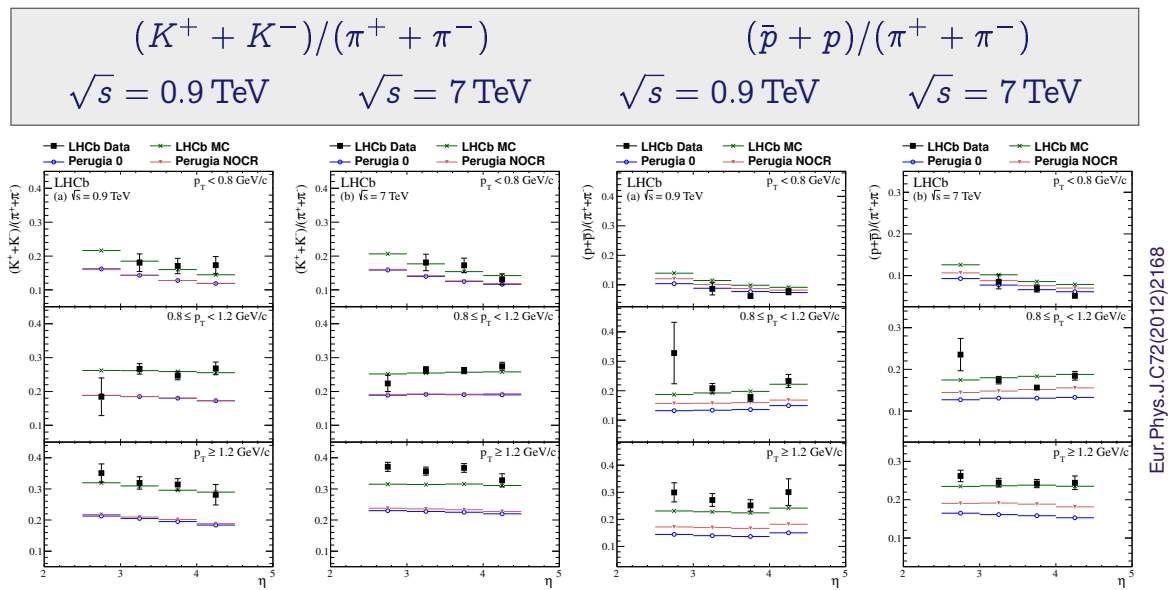

$\rightarrow$ strangeness suppression very similar to baryon suppression

$\rightarrow$ less suppression at larger $p_{T}$

$\rightarrow$ reasonable description only by LHCb-tune of PYTHIA 6 


\section{Lнсb Strange-baryon suppression}

$$
\sqrt{s}=0.9 \mathrm{TeV} \quad \sqrt{s}=7 \mathrm{TeV}
$$
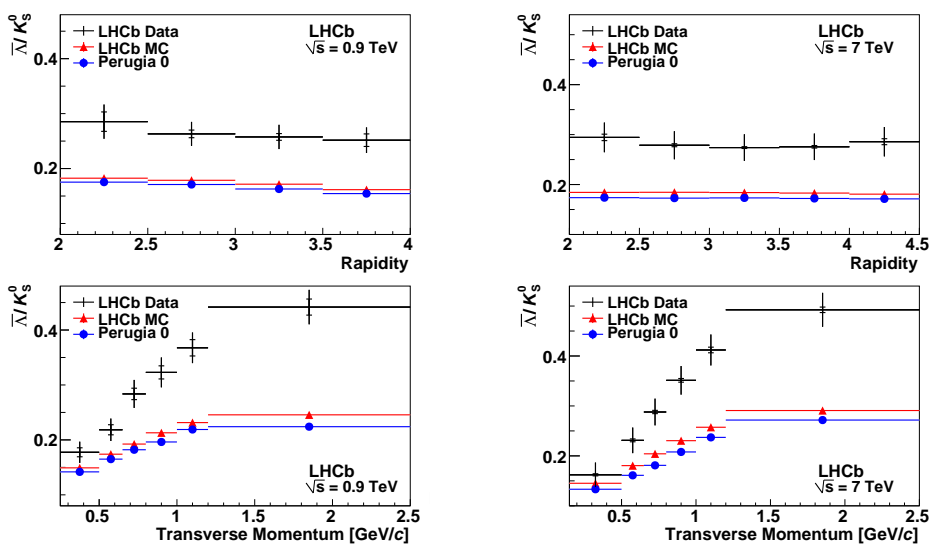

$\rightarrow$ significantly reduced baryon suppression at large $p_{T}$

$\rightarrow$ all considered PYTHIA 6 tunes fail to describe the strangeness-data 


\section{wisch Baryon number transport}

$$
\sqrt{s}=0.9 \mathrm{TeV}
$$

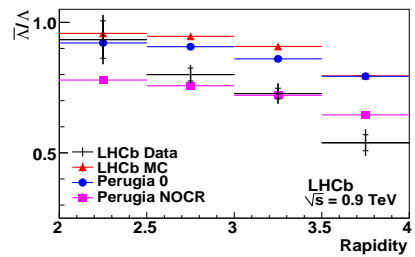

$\sqrt{s}=7 \mathrm{TeV}$

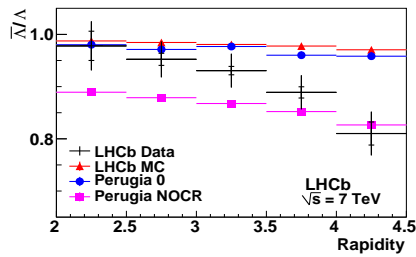

$\square$ all considered PYTHIA 6 tunes fail to describe observed $y$-dependence $\square$ behavior as a function of $\Delta y$ is independent of $\sqrt{s}$ for $\bar{\Lambda} / \Lambda$ and $\bar{p} / p$
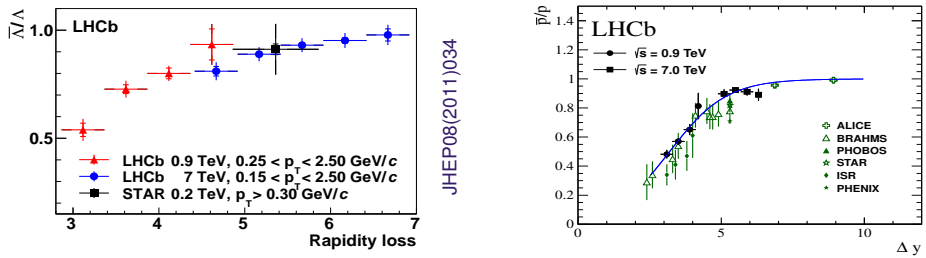


\section{whes $\phi$-meson production}

$\rightarrow$ higher sensitivity to direct strangeness production

$\square$ clean experimental signature $\phi \rightarrow K^{+} K^{-}$
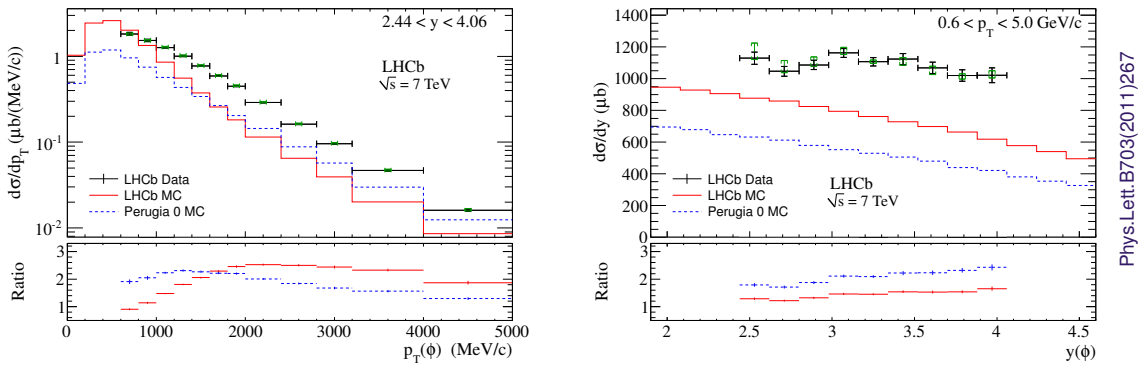

$\rightarrow$ cross-section underestimated by PYTHIA 6 tunes

$\rightarrow$ shapes in $y$ and $p_{T}$ not reproduced by the models

$\rightarrow$ LHCb-tune of PYTHIA 6 describes $K / \pi$ ratio but not $\phi$ cross-section 


\section{HAgeb 4. DRELL-YAN PRODUCTION}

$\rightarrow$ LHCb physics reach

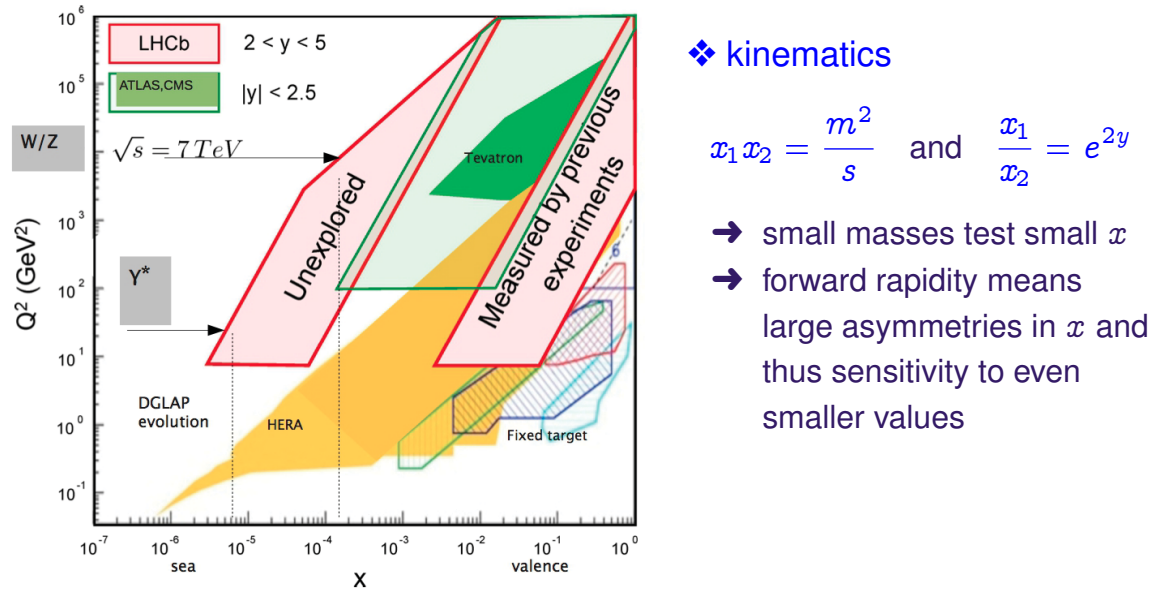

* LHCb allows to probe PDFs in a completely unexplored regime! 


\section{whch Drell-Yan results}

$\rightarrow$ Drell-Yan di-muon cross-section vs invariant mass (LHCb-CONF-2012-013)

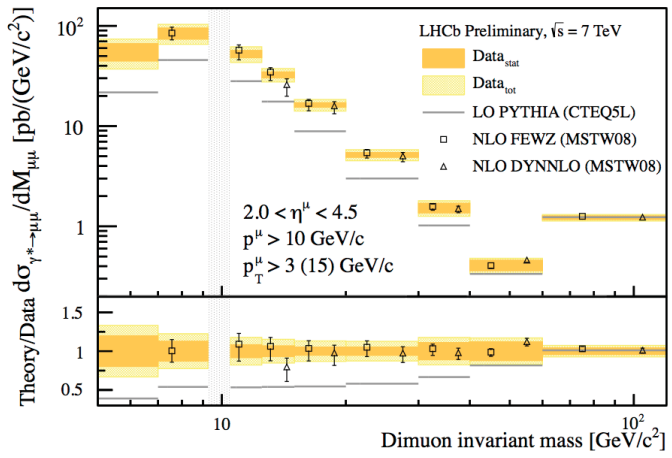

$$
\begin{aligned}
& >q \bar{q} \rightarrow \gamma^{*} \rightarrow \mu^{+} \mu^{-} \\
& >37 \mathrm{pb}^{-1} \text { recorded in } 2010 \\
& >5<M_{\mu \mu}<120 \mathrm{GeV} / c^{2} \\
& >\text { isolated high- } p_{T} \text { muons }
\end{aligned}
$$

$\square$ template-fits to isolation distribution of signal and background

$\square$ leading order predictions systematically too low

$\square$ NLO predictions in good agreement with the data 


\section{thich 5. SUMMARY AND OUTLOOK}

$\rightarrow$ LHCb has a wide QCD-related physics program. . .

$\square$ studies of global event properties

$\rightarrow$ energy flow and multiplicities not well described by PYTHA 6 tunes

$\rightarrow$ cosmic ray models not tuned to HEP data do surprisingly well

$\rightarrow$ better overall description by PYTHIA 8 and Herwig++

$\square$ production measurements for identified particles

$\rightarrow$ antiparticle/particle ratios generally well described

$\rightarrow$ strangeness and baryon suppression too strong in PYTHIA 6 tunes

$\rightarrow$ baryon number transport not described by PYTHIA 6 tunes

$\rightarrow$ baryon number transport with rapidity difference independent of $\sqrt{s}$

$\square$ Drell-Yan production

$\rightarrow$ measurements to masses as low as $5 \mathrm{GeV} / c^{2}$ and $x \sim 10^{-5}$

$\rightarrow$ good agreement with NLO predictions

many results with valuable input for model tuning! 


\section{wich Selected results on forward production}

* global event properties of pp collisions

$\rightarrow$ EPJC73(2013)2421 Measurement of the forward energy flow at $\sqrt{s}=7 \mathrm{TeV}$

$\rightarrow$ EPJC74(2014)2888 Measurement of charged particle multiplicities at $\sqrt{s}=7 \mathrm{TeV}$

* light quarks and strangeness in pp collisions

PLB693(2010)69 Prompt $K_{S}^{0}$ production in pp collisions at $\sqrt{s}=0.9 \mathrm{TeV}$

$\rightarrow$ PLB703(2011)267 Measurement of the inclusive $\phi$ cross-section at $\sqrt{s}=7 \mathrm{TeV}$

$\rightarrow$ JHEP08(2011)034 Measurement of $V^{0}$ production ratios at $\sqrt{s}=0.9$ and $7 \mathrm{TeV}$

$\rightarrow$ EPJC72(2012)2168 Prompt hadron production ratios at $\sqrt{s}=0.9$ and $7 \mathrm{TeV}$

- proton-lead collisions at $\sqrt{s_{\mathrm{NN}}}=5 \mathrm{TeV}$

JHEP02(2014)072 Study of $J / \psi$ production and cold nuclear matter effects open charm and charmonium in pp collisions

NPB871(2013)1

EPJC71(2011)1645

EPJC72(2012)2100

JHEP02(2013)041

JHEP06(2013)064

JPG40(2013)045001
Prompt charm production in pp collisions at $\sqrt{s}=7 \mathrm{TeV}$

$\mathrm{J} / \psi$ production in pp collisions at $\sqrt{s}=7 \mathrm{TeV}$

$\psi(2 S)$ meson production in pp collisions at $\sqrt{s}=7 \mathrm{TeV}$

$J / \psi$ production in pp collisions at $\sqrt{s}=2.76 \mathrm{TeV}$

Production of $J / \psi$ and $\Upsilon$ mesons in at $\sqrt{s}=8 \mathrm{TeV}$

Exclusive $J / \psi$ and $\psi(2 S)$ production at $7 \mathrm{TeV}$ 